\title{
Genome Editing in Stem Cells
}

\author{
Lorna M. FitzPatrick • Tristan R. McKay
}

Published online: 27 January 2015

(C) Springer International Publishing AG 2015

\begin{abstract}
Progress in the derivation, expansion and differentiation of human embryonic stem cells in vitro has led to the development of a valuable platform for studying disease as well as providing an inexhaustible resource for cell replacement therapies. Targeted modification of stem cells utilising synthetic nucleases provides a means to genetically alter the genome in an efficient, site-specific manner. Editing with this approach offers a possibility to improve disease modelling in both patient-derived induced pluripotent stem cells (iPSCs) and embryonic and adult stem cells. It is possible that in the future, patients with monogenic diseases could be treated or cured by transplantation of autologous cells that have been altered to have the corrected genetic sequence. Currently, genetic modification of stem cells in vitro is most often achieved using siRNA or viral transduction; however, this can result in only transient modulation or raise the possibility of insertional mutagenesis, respectively. Targeted genome editing using zinc finger nucleases (ZFNs), transcription activator-like effector nucleases (TALENs) and more recently clustered regularly interspaced short palindromic repeats (CRISPRs)/CRISPR-associated protein 9 (Cas9) have been developed to overcome the drawbacks of gene transfer. In this review, we mechanistically outline these technologies before focusing on their expanding utility within the biomedical sciences.
\end{abstract}

Keywords Genome editing · Gene insertion · Gene disruption · Induced pluripotent stem cells $\cdot$ Cell therapy $\cdot$ Stem cell models

This article is part of the Topical Collection on Genome Editing

L. M. FitzPatrick $(\bowtie) \cdot$ T. R. McKay

Stem Cell Research Group, Molecular Cell Sciences Research

Centre, Cardiovascular and Cell Sciences Research Institute,

St. George's University of London, Cranmer Terrace,

London SW17 0RE, UK

e-mail: p1207314@sgul.ac.uk

\section{Introduction: Mechanisms of Genome Editing}

Targeted nuclease technology is dependent on the use of engineered molecules containing a sequence-specific DNA binding domain fused to a non-specific Fokl endonuclease enzyme that must dimerize for efficient site-specific cleavage. When applied, a double-strand break occurs in the DNA, and the break is repaired by conventional DNA repair mechanisms: non-homologous end joining (NHEJ) or homologous recombination (HR). $\mathrm{C}_{2} \mathrm{H}_{2}$ zinc fingers (ZF), originally described in Xenopus laevis [1], are one of the most common types of DNA binding motifs found in the human genome and are considered the first generation of engineered nuclease DNA binding domains. They are approximately 30 amino acids in length, and each architecturally simple domain (a $\beta \beta \alpha$-fold) has the capacity to bind to 3 base pairs of DNA [1]. To overcome problems that may arise from ZF targeting non-unique sequences, a number of groups have worked on developing polydactyl ZF proteins utilising a linker sequence to tether multiple ZF together, binding up to 18 base pairs of DNA. This confers specificity in a genome of up to 68 billion base pairs [2-4]. Furthermore, Maeder et al. developed oligomerized pool engineering ('OPEN'), a publicly available highly efficient approach to design multi-finger arrays that can be customised to a DNA fragment of choice [5].

Fifteen years later, an alternative second-generation genome editing platform to ZFNs was established and rapidly came to prominence in the literature. Similar to ZF proteins, TALENs consist of modular DNA binding motifs that target contiguous DNA sequences and are also linked to the Fokl enzyme to execute site-specific double-strand breaks. However, these proteins, naturally found in plant pathogenic bacteria Xanthomonas, are considered to be more accessible to researchers aiming to engineer innovative fusion proteins as a tool for altering the genome [6]. The DNA binding motif of 
transcription activator-like (TAL) effector proteins consist of $\sim 35$ tandem repeats, and each repeat (consisting of two amino acids) appears to be specific for a single nucleotide [6]. Therefore designing TALENs is more straightforward than ZFNs whilst maintaining similar diversity in sequence design. Moreover, when generating TALENs, there is no requirement for re-engineering the linker or spacer regions between the residues as is necessary when producing ZFNs. Consequently, TALEN production is faster, more economically viable and highly specific. Several plasmid sets are now openly available from repositories (www.addgene.org/) to aid the development of desirable fusion molecules including fast ligation-based automatable solid-phase high-throughput (FLASH) [7], plasmid-based methods such as 'TAL Plasmids Sequence Assembly Tool' (Golden Gate) [8] and ligation-independent cloning [9].

The most recent, and arguably most exciting, method for genome editing, the CRISPR/Cas system, was originally described in a broad spectrum of bacteria and archaea as an RNA-mediated defence mechanism via genetic silencing [10]. Collectively, a number of research groups [11-13] deciphered the mechanism and ultimately demonstrated that it could be manipulated to direct sequence-specific DNA cleavage in many model systems including zebrafish [14, 15], mouse [16, 17] and human [18, 19]. The CRISPR/Cas pathway is akin to the RNA interference pathway in eukaryotes as both methods recognise long RNA precursors that are further processed to hinder translation. In the CRISPR system, a guide RNA (gRNA) sequence can be designed to target the genomic locus of choice. Directly following the target sequence is a protospacer adjacent motif (PAM). PAM, along with a seed sequence contained within the gRNA, allows for stable and efficient DNA binding causing a double-strand break mediated by the Cas endonuclease enzyme (Cas9 is most commonly used) [20]. The CRISPR/Cas9-based technology makes genome editing fast (desirable target gRNA plasmids are easily produced), simple and relatively cheap; many component plasmids are also available from freely available repositories. Mali et al. showed that CRISPR/Cas9 genome editing was more efficient than TALENs and ZFNs in combination with the ease of retargeting the system to another locus in the genome [18]. However, the major caveat to this speedy technology is that the target RNA sequence is shorter than those of TALENs and ZFN; thus, it is more difficult to prevent off-target genomic disruption.

DNA cleavage either by Cas9 or Fokl results in a doublestrand break, inducing activation of internal DNA repair mechanism to preserve genomic integrity. The two most common mechanisms by which DNA is religated are NHEJ or homologous recombination. If repair is carried out by NHEJ, the $\mathrm{Ku} 70 / \mathrm{Ku} 80$ end-binding protein complex religates the DNA. However, this repair mechanism is executed in an error-prone manner, often resulting in insertions, deletions
Fig. 1 Mechanisms of genome editing by ZFNs, TALENs and CRISPR/ Cas9. a ZFN bind to target DNA (one zinc finger to $3 \mathrm{bp}$ of DNA) where the Fokl endonuclease enzyme dimerizes to induce a non-specific double-strand break. TALENs behave in a similar manner to ZFs; however, one TALEN binds to one base pair of DNA. The CRISPR guide RNA binds to the target DNA and is cleaved by the Cas9 endonuclease enzyme, aided by PAM sequences and a 'seed sequence'. b The resulting cleaved DNA can undergo repair by two cellular mechanisms, NHEJ or homologous recombination. c NHEJ is error prone so often introduces 'indels'. Homologous recombination can be manipulated to insert a transgene, provided the transgene contains flanking recombination sites

and substitutions at the break site [21]. This strategy is exploited during genome editing to mutate or silence target genes. For gene insertion and correction, homologous recombination is manipulated to incorporate donor DNA (most likely a gene) exogenously introduced to the cell on a separate construct, provided the flanking regions contain recombination sites (Fig. 1).

\section{Applications of Genome Editing in Stem Cells}

\section{Gene Insertion}

There is great potential to treat human monogenic diseases such as spinal muscular atrophy, sickle cell and X-linked adrenoleukodystrophy (X-ALD) by gene complementation or correction. Gene therapy has been used widely to treat monogenic diseases and there have been many recent successes [22-25]. Achieving widespread gene delivery to ameliorate disease is a major challenge beyond proof of concept and has largely been based on improving gene delivery vector technology and long-term transgene expression. To date, some of the most effective gene therapy approaches have involved ex vivo gene therapy of blood cells to treat Wiskott-Aldrich syndrome (WAS) [26], X-linked severe combined immunodeficiency (X-SCID) and adenosine deaminase deficiency (ADA) [27, 28]. X-SCID is caused by mutations in the IL2RG gene situated on the X chromosomal at Xq13.1 and affects males by causing defective maturation of $T$ cells and NK cells resulting in severe immunodeficiency. Hacein-BeyAbina et al. showed that an ex vivo gene complementation strategy using retroviral-mediated gene therapy targeting haematopoietic stem cells resulted in long-term phenotypic correction [27]. Although most patients received therapeutic benefit, two developed leukaemia due to insertional mutagenesis; a nearby oncogene (LMO2) became deregulated as a result of random integration [29]. The occurrence of leukaemia was not isolated to the X-SCID patients treated in this study. Between 1999 and 2009, 5 of the 20 participants across 5 independent X-SCID gene therapy trials in Europe developed leukaemia due enhancer activity of the provirus inserted 
a

\section{ZFN}

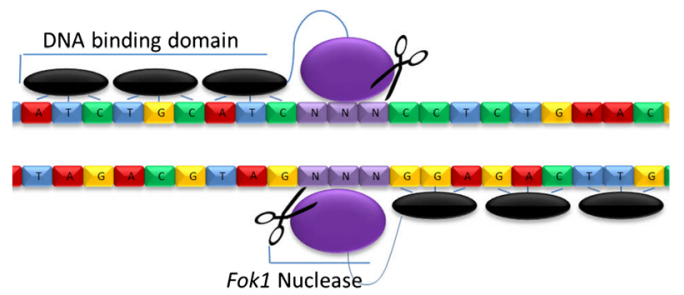

TALEN
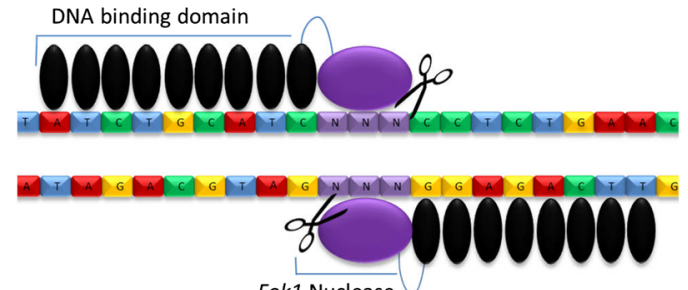

\section{CRISPR/Cas9}

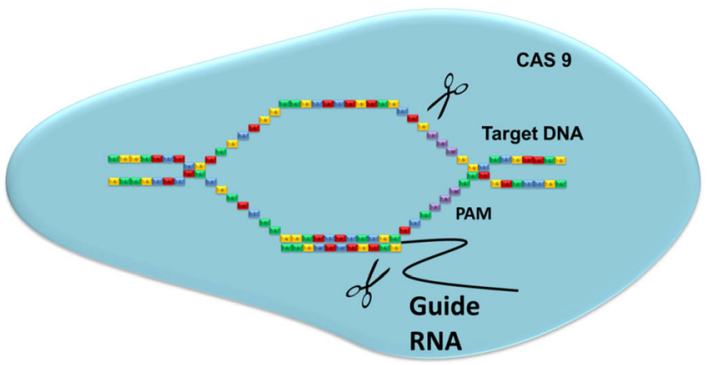

b

\section{Double-strand break}

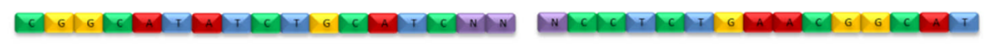

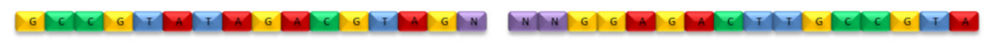

C

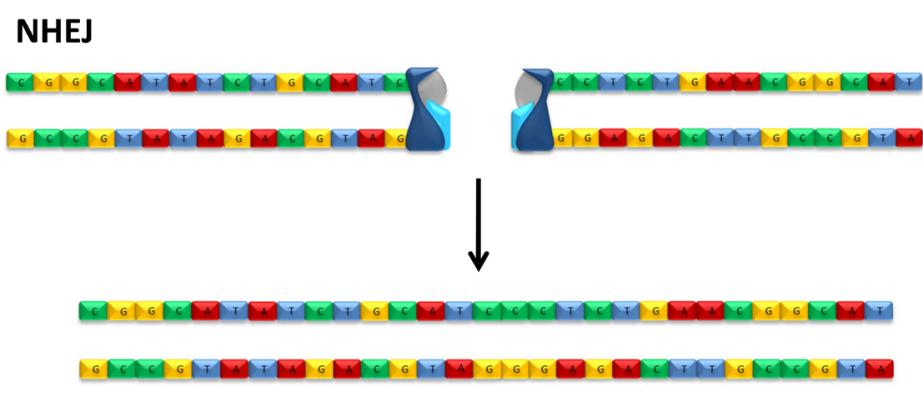

\section{Homologous recombination}

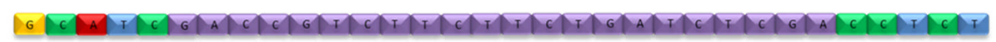

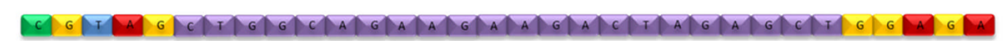

$\mathrm{HR}>$

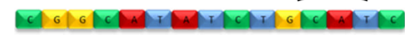

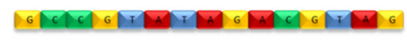

$>\mathrm{HR}_{\mathrm{R}}$

CITICI G

G)

$\downarrow$

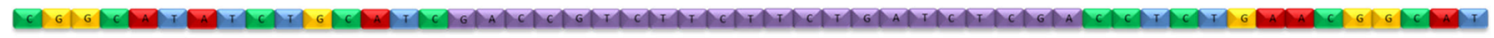

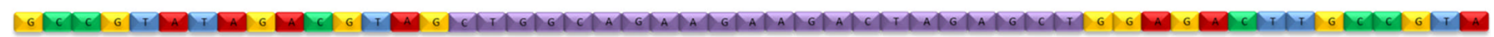

into an oncogenic gene region [30]. In the WAS trial, most patients also developed leukaemia [26]. Notwithstanding oncogene deregulation associated with non-specific genomic integration, there are other impediments to this method of 
gene transfer; host immune responses may counteract the benefit conferred with transgene expression and it is not uncommon to encounter long-term transgene silencing as a result of promoter methylation [31]. Artificial nucleasemediated genome editing could potentially alleviate viral vector-associated risks. Moreover, the levels and frequency of expression of the transgene are under the regulation of the endogenous promoter, so the level of functional protein produced should be equivalent to that of non-patient controls [32]. In 2005, Urnov et al. successfully used ZFNs to target and cleave the endogenous IL2RG gene in cells in vitro [33]. Two years later, Lombardo et al. combined the infectious properties of an integration-deficient lentiviral vectors (IDLV) with the site-specific properties of ZFN to increase the efficiency (13-39 \% across different cell types) of DNA insertions at the same locus. A green fluorescent protein (GFP) reporter contained within the homology arms of the ILR2G donor sequence was co-transduced with the ZFNexpressing IDLV, and PCR analysis of the $\mathrm{GFP}^{+}$cells sorted by fluorescence-activated cell sorting (FACS) showed the anticipated integration junctions between the cassette and the ILR2G locus [34]. In the same study, the authors also implemented this system to insert GFP into the CCR5 locus of cord blood $\mathrm{CD} 34^{+}$haematopoietic progenitor cells and two human embryonic stem (ES) cell lines, achieving gene targeting at higher levels than previously observed in human stem cells. CCR5 is a chemokine receptor found on the surface of white blood cells that acts as a co-receptor to HIV-1, facilitating viral entry into the host. Individuals carrying a 32-bp deletion within the coding region produce a non-functional receptor and are subsequently resistant to HIV-1 infection [35].

A follow-up study also by the Naldini group in 2014 further supports the clinical translation of ZFN-based correction of monogenic disorders. Using the same IDLV/ZFN system, haematopoietic stem cells (HSCs) from the bone marrow of a 4-month-old X-SCID patient were modified to incorporate the fully functional IL2RG gene, in vitro. They saw long-term reconstitution of IL2RG expression in the lymphoid progeny of the HSCs [36]. This exciting approach has great clinical potential if integration-deficient lentiviral vectors pass relevant safety assessments, and the appropriate stem cell population can be isolated from patients for modification.

\section{Gene Disruption}

Although introducing a transgene via HR is an excellent way to exploit the cell internal repair mechanisms to deliver therapeutic benefit, recombination efficiency is poor and may vary with regard to the cell type. It is thought that HR events are dependent on the cell cycle, preferentially occurring in the late $\mathrm{S}, \mathrm{G}_{2}$ and $\mathrm{M}$ stages [37]. In the absence of a donor plasmid for HR, NHEJ is the dominant method for double-strand break repair. Hans Clevers' group optimised gene disruption by NHEJ via the CRISPR/Cas9 system in murine and human intestinal stem cells in 2013. They used a short guide RNA to target the $A P C$ gene - a negative regulator of the Wnt pathway-and found that only stem cells in which biallelic inactivation occurred had the capacity to proliferate in the absence of the Wnt agonist R-spondin [38].

In 2008 , Perez et al. permanently modified the HIV coreceptor CCR5 in $\mathrm{CD} 4{ }^{+} \mathrm{T}$ cells by designing ZFNs to target the first transmembrane domain of the gene and allowing NHEJ mechanisms to introduce random mutations. They noted that $30 \%$ of all sequences analysed had a 5 -bp insertionduplication of the region between the ZFN binding sites [39]. However, they also observed a wide range of other deletion and insertion mutations at the break site. So, although this paper shows great potential to impart HIV-1 resistance in T lymphocytes or bone marrow haematopoietic stem cells from patients, translation to the clinic as a therapy may be hindered by the unpredictable nature of NHEJ repair as a tool for gene disruption.

Designing an artificial nuclease with a recognition sequence targeted to a locus, where the only difference between the endogenous sequence and the disruption donor sequence is the exact bases intended for modification, would allow for the introduction of missense or nonsense mutations in a regulated manner. In recent years, there has been a shift towards this type of genome editing, particularly in the context of pluripotent stem cells.

\section{Genome Editing in Pluripotent Stem Cells}

The pioneering work of Yamanaka and co-workers on somatic reprogramming and induction of pluripotency by the forced expression of four transcription factors (Oct4, Sox2, Klf4 and $c-M y c)$ [40] allows for iPSC lines to be generated from patient-derived fibroblasts. This methodology has led to the establishment of a plethora of disease-specific human iPSC lines from patients [41-44]. Targeted differentiation of such iPSC means that the biology of disease can be studied in patient-derived cell types that would otherwise be impossible to obtain, such as those of the central nervous system (CNS) and heart $[45,46]$. This remarkable research platform now facilitates the unification of genomics, transcriptomics and biochemistry in disease relevant cells in the lab. Last year, a breakthrough research article was published by Jiang et al. where the authors used ZFNs to model Down's syndrome (trisomy 21). The study demonstrated that it was possible to correct the chromosome 21 gene dosage imbalance by recombining a single non-coding RNA, XIST (X-inactivation gene), into the DYRK1a locus (21q22) [47•]. XIST is responsible for compensating for the difference in dosage of Xlinked genes between males (XY) and females (XX). Located on the inactive $\mathrm{X}$ chromosome, the non-coding RNA 
accumulates across the chromosome, dampening gene activity [48]. One of the remarkable achievements of this study is the size of the doxycycline-controlled transgene that was targeted to the locus of male patient-derived iPSC - $21 \mathrm{~kb}$ of transgene was successfully integrated along with the doxycycline control construct in an allele-specific manner. This is the largest transgene to date to be integrated in a site-specific manner. In nearly all clones $(98.5 \%)$ positive for XIST RNA, the insertion was localised to DYRK1A, and high-resolution cytogenetic banding on 6 chosen clones suggested that significant abnormalities were found only on chromosome 21 . Within 5 days of XIST activation, the inactivated chromosome developed into a Barr body, carrying repressive histone markings, and analysis of chromosome 21 in nuclei showed that the DNA became markedly condensed. In addition, 3 weeks after induction, $97 \%$ of $\mathrm{CpG}$ island containing genes on chromosome 21 demonstrated increased promoter methylation. Notably, when the pluripotent stem cells were subjected to neural induction culture conditions, the XIST clones had the capacity to develop into rosettes, a hallmark of multipotent neural stem cells. The uninduced lines, however, showed a delay in rosette formation when compared to the corrected counterparts. This ZFN-based study provides an excellent platform therefore to examine a number of developmental and cellular aberrations in Down's syndrome patients [47].

Recent iPSC studies comparing genetics and biochemistry, particularly in complex multifactorial diseases, have been somewhat compromised by correlating patients with normal or heterozygote siblings as the sample genomes are not identical [49]. Furthermore, research in both mouse [50, 51] and human $[52,53]$ models also suggest that early iPSCs retain some epigenetic memory from the cells that they originate from, and the differentiation capacity of the iPSCs is often biassed [50-53]. An individual gene mutation's specific contribution to disease can only realistically be evaluated in comparison to a truly isogenic control. This can be achieved using artificial nuclease technology to revert the known mutation back to normal; thus, the only genetic difference between the lines is the disease-associated mutation. Designing an artificial nuclease with a recognition sequence targeted to a locus, where the only difference between the endogenous sequence and the disruption donor sequence is the exact bases intended for modification, allows for the introduction of missense or nonsense mutations in a regulated manner. Such approaches are particularly useful for diseases that are late onset and/or manifest discrete functional or morphological changes when cultured in vitro. An et al. reported a study employing the CRISPR-based genome editing strategy to model Huntington's disease (HD) in patient-derived iPS cells [54]. Homologous recombination was performed to produce a $\mathrm{HD}$ isogenic allelic series using two Cas9 enzymes, wild-type and Cas9 D10A, and found that the mutant version reduced the number of offtarget events associated with the wild-type enzyme.

In a reversal of the isogenic control principle, $\mathrm{hES}$ cell lines generated from genetically normal embryos can be subjected to disease-causing mutagenesis using genome editing. In 2011, Soldner et al. performed a simple but elegant ZFN study to introduce two point mutations (A53T/E46K) in the $\alpha$ synuclein gene in hES cells, generating a Parkinson's disease model. Conversely, the group then repaired the A53T mutation in patient-derived iPSCs, successfully altering one base pair only, without altering the rest of the genome [55•]. To do this, as described above, an exogenous double- or singlestranded sequence of template DNA was introduced with the locus-specific mutations to serve as a template for DNA repair by homologous recombination. More recently, Ding et al.

Table 1 Comparison of ZFN, TALEN and CRISPR/Cas technologies

\begin{tabular}{|c|c|c|}
\hline Technology & Advantages & Disadvantages \\
\hline ZFN & $\begin{array}{l}\text {-Commercially available for academic research from Sigma-Aldrich } \\
\text {-Many proteins already available } \\
\text {-Publicly available strategy, OPEN, to aid with ZFN construction } \\
\text {-Efficient delivery of large transgenes } \\
\text {-Progress made in translation to the clinic. Sangamo have } \\
\text { ZFNs in phase } 1 \text { and phase } 2 \text { trials to treat HIV-1 by } \\
\text { targeting the CCR5 gene }\end{array}$ & $\begin{array}{l}\text {-Expensive to purchase } \\
\text {-Difficult to construct if not obtained commercially } \\
\text {-Depending on the target sequence, some off-targeting } \\
\text { may occur }\end{array}$ \\
\hline TALEN & $\begin{array}{l}\text {-Unlike ZFNs, TALEN production does not require re-engineering } \\
\text { of the linkage between molecules } \\
\text {-Rapid assembly using Golden Gate cloning, high throughput } \\
\text { solid phase assembly and ligation-independent cloning } \\
\text {-The ease of production allows for genome editing at multiple } \\
\text { target sites in a cell } \\
\text {-Fewer reported off-target events compared to CRISPR }\end{array}$ & $\begin{array}{l}\text {-Delivery can be more difficult as TALENs are large } \\
\text { proteins } \\
\text {-Some sensitivity to methylation }\end{array}$ \\
\hline CRISPR/Cas9 & $\begin{array}{l}\text {-Easy to design and highly versatile } \\
\text {-Relatively cheap } \\
\text {-Paired single-strand break mutants (nickases) have recently been } \\
\text { developed to reduce off-target effects of CRISPRs } \\
\text {-High frequency of editing achieved }\end{array}$ & $\begin{array}{l}\text {-Off-target effects at high frequency have been } \\
\text { recorded [63] as the target sequence of CRISPRs is } \\
\text { generally shorter than ZFN and TALENs } \\
\text {-Some target design constraints (e.g. a GG sequence } \\
\text { must directly succeed the short guide RNA target) }\end{array}$ \\
\hline
\end{tabular}


established a swift and efficient TALEN assembly platform and successfully re-engineered the genome of $2 \mathrm{hES}$ cell lines, generating mutant alleles of 15 different genes, such as $A K T 2$, CFTR and PLIN1 [56]. In one example, they demonstrated that it was possible to determine functional effects of a modified SORT1 gene in hES-derived hepatocyte-like cells. They also observed phenotypic changes in the other mutant lines generated (when compared to the isogenic control lines) that were directly linked to a number of pathologies when subjected to differentiation conditions including insulin resistance, lipodystrophy and hypoglycaemia [56].

An added advantage to genome editing over HR is that a selection or reporter cassette can be introduced in conjunction with the desired mutation to provide a means for isolating the modified cell clones. Chen et al. used a lox-P-flanked neomycin cassette when using TALENs to introduce point mutations introduced into the SCN1A gene responsible for causing severe mycological epilepsy of infancy (SMEI) [57]. The selectable marker was excised after generation, selection and characterisation of iPSCs lines. There are many strategically similar studies in iPSCs that use genome editing to either correct or disrupt a gene by introducing a point mutation via artificial nuclease technology, providing new models for studying diseases such as Barth syndrome, HIV and $\beta$ - thalassemia [58-60]. The technology is also becoming more innovative; research groups are combining the more desirable aspects of each artificial nuclease to further increase efficiency and develop highly customised $\mathrm{iPSC} / \mathrm{hESC}$ lines [60-62]. The cutting edge approach by Gonzales et al. produced a TALEN-mediated Cas9 inducible hES cell line and then used CRISPRs for biallelic targeting of six different genes, thus producing a double and triple knockout line [61]. The authors of the above studies all stressed the rapidity with which the lines were generated. Isolating patient samples for reprogramming into an embryoniclike stem cell can be both time consuming and costly. Furthermore, multiplexed targeting such as this may provide a novel platform to study diseases that are polygenic for up to dozens of gene, rather than being restricted to treating monogenic disorders using viral vectors containing a single transgene.

\section{Conclusion: Summary and Future Directions}

To conclude, it is clear that novel genome editing technologies will dramatically aid in the progression of cell-based therapy and in our understanding, of many genetically linked diseases. As the field has expanded dramatically in recent years, it is now possible to generate permanent and specific multiple-hit pluripotent cell lines for differentiation into the disease-associated cell type. However, one of the limiting factors in this respect is the availability of well-established specification protocols. Another confining factor, particularly crucial when considering translation into the clinic, is the need for highly efficient and sometimes biallelic editing events. Although there are few studies done to directly compare the relative efficiencies of ZFNs, TALENs and CRISPRS/Cas9, one research group, Ding et al., surmised that the CRISPR/Cas9 system is superior to TALEN-mediated genome editing in human pluripotent stem cells, possibly as a result of increased tolerance to the Cas9 enzyme [64]. Combination approaches are sometimes favoured over more traditional methods to increase efficiency such as transposon/CRISPR [60], TALEN/CRISPR (iCRISPR) [61] and IDLV/ZFN $[34,36]$ as mentioned. Table 1 outlines both the advantages and disadvantages of current ZFN, TALEN and CRISPR/Cas 9 technology.

To provide therapeutic benefit to patients, genome editing strategies to correct disease-linked mutations in either adult or iPSC-derived progenitor cells for autologous transplant must be deemed safe, i.e. not disrupt other loci in the genome. Excitingly, a whole genome analysis study by Veres et al. demonstrated that there is a very low incidence of off-target mutation events induced by both TALEN and CRISPR/Cas9 targeted to the SORT1 gene in hES cells [65•]. Currently, there is encouraging progress being made in gene correction for a variety of diseases. For example, Schwank et al. have recently published data that provides evidence of functional repair of the CFTR gene in adult stem cells isolated from cystic fibrosis patients, mediated by the CRISPR/Cas9 system. They also observed very few off-target effects associated with the treatment. Moreover, a forskolin-response assay implied that the function of the disease-associated transmembrane receptor was restored [38]. Although this disease affects multiple organs and so would be complex to treat using this method, this research is indicative of the potential for genome editing to advance cell replacement therapy.

\section{Compliance with Ethics Guidelines}

Conflict of Interest Lorna M. FitzPatrick and Tristan R. McKay declare that they have no conflict of interest.

Human and Animal Rights and Informed Consent This article does not contain any previously unpublished studies with human or animal subjects performed by any of the authors.

\section{References}

Papers of particular interest, published recently, have been highlighted as:

- Of importance

1. Miller J, McLachlan AD, Klug A. Repetitive zinc-binding domains in the protein transcription factor IIIA from Xenopus oocytes. EMBO J. 1985;4:1609-14. 
2. Beerli RR, Barbas 3rd CF. Engineering polydactyl zinc-finger transcription factors. Nat Biotechnol. 2002;20:135-41.

3. Liu Q, Segal DJ, Ghiara JB, Barbas 3rd CF, et al. Design of polydactyl zinc-finger proteins for unique addressing within complex genomes. Proc Natl Acad Sci U S A. 1997;94(11):5525-30.

4. Moore M, Klug A, Choo Y. Improved DNA binding specificity from polyzinc finger peptides by using strings of two-finger units. Proc Natl Acad Sci U S A. 2001;98:1437-41.

5. Maeder ML, Thibodeau-Beganny S, Osiak A, et al. Rapid "open source" engineering of customised zinc-finger nucleases for highly efficient gene modification. Mol Cell. 2008;31(2):294-301.

6. Boch J, Scholze H, Schornack S, et al. Breaking the code of DNA binding specificity of TAL-type III effectors. Science. 2009;326(5959):1509-12.

7. Reyon D, Tsai SQ, Khayter C, et al. FLASH assembly of TALENs for high-throughput genome editing. Nat Biotechnol. 2012;30: 460-5.

8. Engler C, Kandiza R, Marillonnet S. A one pot, one step, precision cloning method with high throughput capability. PLoS One. 2008;3(11):e3647. doi:10.1371/journal.pone.0003647.

9. Schmidt-Burgk JL, Kaiser V, Höning K, et al. A ligationindependent cloning technique for high throughput assembly of transcription activator-like effector genes. Nat Biotechnol. 2012;31(1):16-81.

10. Wiedenheft B, Sternberg SH, Doudna JA. RNA-guided genetic silencing systems in bacteria and archaea. Nature. 2012;482:331-8.

11. Makarova KS, Grishin NV, Shabalina SA, et al. A putative RNAinterference-based immune system in prokaryotes: computational analysis of the predicted enzymatic machinery, functional analogies with eukaryotic RNAi, and hypothetical mechanisms of action. Biol Direct. 2006;16:1-7.

12. Barrangou R, Fremaux C, Deveau H, et al. CRISPR provides acquired resistance against viruses in prokaryotes. Science. 2007;315(5819):1709-12.

13. Jinek M, Chylinski K, Fonfara I. A programmable dual-RNAguided DNA endonuclease in adaptive bacterial immunity. Science. 2012;33(9096):816-21.

14. Hwang W, Fu Y, Reyon D, et al. Efficient genome editing in zebrafish using a CRISPR-Cas system. Nat Biotechnol. 2013;31: 227-9.

15. Hruscha A, Krawitz P, Rechenberg A, et al. Efficient CRISPR/Cas9 genome editing with low off target effects in zebrafish. Development. 2013;140:4962-87.

16. Wang H, Yang H, Shivalila CS, Dawlaty M, Cheng A, et al. Onestep generation of mice carrying mutations in multiple genes by CRISPR/Cas-mediated genome engineering. Cell. 2013;153(4): 910-918.

17. Platt R, Chen S, Zhou Y, et al. CRISPR-Cas9 knockin mice for genome editing and cancer modelling. Cell. 2014;159(2):440-55.

18. Mali P, Yang L, Esvelt K, et al. RNA-guided human genome engineering via Cas9. Science. 2013;339(6121):823-6.

19. Sakuma T, Nishikawa A, Kume S, et al. Multiplex genome engineering in human cells using all-in-one CRISPR/Cas9 vector system. Sci Rep. 2014;4:5400.

20. Semenova E, Jore MM, Datsenko KA, et al. Interference by clustered regularly interspaced short palindromic repeat (CRISPR) RNA is governed by a seed sequence. Proc Natl Acad Sci U S A. 2011;108:10098-103.

21. Iliakis G, Wang H, Perrault AR, et al. Mechanisms of DNA double strand break repair and chromosome aberration formation. Cytogenet Genome Res. 2004;104(1-4):14-20.

22. Nathwani AC, Tuddenham EGD, Rangarajan S, et al. Adenovirusassociated virus vector-mediated gene transfer in haemophilia B. N Engl J Med. 2011;365:2357-65.
23. Cartier N, Hacein-Bay-Abina S, Bartholomae CC, et al. Hematopoietic stem cell gene therapy with a lentiviral vector in X-linked adrenoleukodystrophy. Science. 2009;326:818-23.

24. Cavazzana-Calvo M, Payen E, Negre O, et al. Transfusion independence and HMGA2 activation after gene therapy of human $\beta$ thalassaemia. Nature. 2010;467:318-22.

25. Ott MG, Schmidt M, Schwarzwaelder K, et al. Correction of Xlinked chronic granulomatous disease by gene therapy, augmented by insertional activation of MDS1-EVI1, PRDM16 or SETBP1. Nat Med. 2006;12:401-9.

26. Braun CJ, Boztug K, Paurzynski A, et al. Gene therapy for WiskottAldrich syndrome-long term efficacy and genotoxicity. Sci Transl Med. 2014;6:227ra223.

27. Hacein-Bey-Abina S, Le Deist F, Carlier F, et al. Sustained correction of X-linked severe combined immunodeficiency by ex vivo gene therapy. N Engl J Med. 2002;346:1185-93.

28. Aiuti A, Cattaneo F, Galimberti S, et al. Gene therapy for immunodeficiency due to adenosine deaminase deficiency. N Engl Med. 2009;360:447-58.

29. Hacein-Bey-Abina S, Von Kalle C, Schmidt M, et al. LMO2associated clonal T-cell proliferation in two patients after gene therapy for SCID-X1. Science. 2003;302:415-9.

30. Fischer A, Hascein-Bey-Abina S, Cavazzana-Calvo M. 20 years of gene therapy for SCID. Nat Immunol. 2010;11:457-60.

31. Thomas CE, Ehrhardt A, Kay MA. Progress and problems with the use of viral vectors for gene therapy. Nat Rev Genet. 2003;4: 346-58.

32. Porteus $\mathrm{MH}$, Carroll D. Gene targeting using zinc finger nucleases. Nat Biotechnol. 2005;23(8):967-73.

33. Urnov FD, Miller JC, Lee Y, et al. Highly efficient endogenous human gene correction using designed zinc finger nucleases. Nature. 2005;435:646-51.

34. Lombardo A, Genovese P, Beausejour CM, et al. Gene editing in human stem cells using zinc finger nucleases and integrasedefective lentiviral vector delivery. Nat Biotechnol. 2007;25(11): 1298-306.

35. Samson M, Libert F, Doranz B, et al. Resistance to HIV-1 infection in Caucasian individuals bearing mutant alleles of the CCr-5 chemokine receptor gene. Nature. 1996;382:722-5.

36. Genovese P, Schiroli G, Escobar G, et al. Targeted genome editing in human repopulating haematopoietic stem cells. Nature. 2014;510:235-40.

37. Saleh-Gohari N, Helleday T. Conservative homologous recombination preferentially repairs DNA double-strand breaks in the S phase of the cell cycle in human cells. Nucleic Acids Res. 2004;32(12):3683-8

38. Schwank G, Koo BK, Sasselli V, et al. Functional repair of CFTR by CRISPR/Cas9 in intestinal stem cell organoids of cystic fibrosis patients. Cell Stem Cell. 2013;13:653-8.

39. Perez E, Wang J, Miller J, et al. Establishment of HIV-1 resistance in CD4+ T cells by genome editing using zinc-finger nucleases. Nat Biotechnol. 2008;26(7):808-16.

40. Takahashi K, Yamanaka S. Induction of pluripotent stem cells from mouse embryonic fibroblasts and adult fibroblast cultures by defined factors. Cell. 2006;126(4):663-76.

41. Dimos JT, Rodolfa KT, Niakan KK, et al. Induced pluripotent stem cells generated from patients with ALS can be differentiated into motor neurons. Science. 2008;321:1218-21.

42. Park IH, Arora N, Huo H, et al. Disease-specific induced pluripotent stem cells. Cell. 2008;134:877-86.

43. Ye Z, Zhan H, Mali P, et al. Human induced pluripotent stem cells from blood cells of healthy donors and patients with acquired blood disorders. Blood. 2009;114:5473-80.

44. Raya A, Rodriguez-Piza I, Guenechea G, et al. Disease-corrected haematopoietic progenitors from Fanconi anaemia induced pluripotent stem cells. Nature. 2009;460:53-9. 
45. Ebert $\mathrm{AD}, \mathrm{Yu} \mathrm{J}$, Rose Jr FF, et al. Induced pluripotent stem cells from a spinal muscular atrophy patient. Nature. 2009;457:277-80.

46. Moretti A, Laugwitz KL, Dorn T, et al. Pluripotent stem cell models of human heart disease. Cold Spring Harb Perspect Med. 2013;3: a014027.

47. Jiang J, Jing Y, Cost G, et al. Translating dosage compensation to trisomy 21. Nature. 2013;500:296-302. Uses ZFN technology to introduce a very large transgene to correct Trisomy 21 .

48. Brown CJ, Hendrich BD, Rupert JL, et al. The human XIST gene: analysis of a $17 \mathrm{~kb}$ inactive $\mathrm{X}$-specific RNA that contains conserved repeats and is highly localised within the nucleus. Cell. 1992;71: $527-42$.

49. Musunuru K. Genome editing of human pluripotent stem cells to generate human cellular disease models. Dis Model Mech. 2013;6(4):896-904.

50. Kim K, Doi B, Ng K, et al. Epigenetic memory in induced pluripotent stem cells. Nature. 2010;467:285-90.

51. Polo JM, Liu S, Figueroa ME, et al. Cell type of origin influences the molecular and functional properties of mouse induced pluripotent stem cells. Nat Biotechnol. 2010;28:848-55.

52. Bar-Nur O, Russ HA, Efrat S, et al. Epigenetic memory and preferential lineage-specific differentiation in induced pluripotent stem cells derived from human pancreatic islet beta cells. Cell Stem Cell. 2012;9(1):17-23.

53. Marchett $\mathrm{M}$, Yeo G, Kainohana $\mathrm{O}$, et al. Transcriptional signature and memory retention of human-induced pluripotent stem cell. PLoS One. 2009;4(9):e7076.

54. An MC, O'Brien RN, Zhang N, et al. Polyglutamine disease modelling: epitope based screen for homologous recombination using CRISPR/Cas9 system. PLOS Curr Huntingt Dis. 2014;1:1-19.

55. Soldner F, Laganiere J, Cheng A, et al. Generation of isogenic pluripotent stem cells differing exclusively at two early onset Parkinson's point mutations. Cell. 2011;146(2):318-31. This paper generates isogenic iPSC lines using ZFN to model Parkinson's.
56. Ding Q, Lee YK, Schaefer EAK, et al. A TALEN genome editing system for generating human stem cell-based disease models. Cell Stem Cell. 2013;12:238-51.

57. Chen W, Liu J, Zhang L, et al. Generation of the SCN1A epilepsy mutation in hIPS cells using the TALEN technique. Sci Rep. 2014;4:5404.

58. Zweigerdt R, Gruh I, Martin U. Your heart on a chip: iPSC-based modeling of Barth-syndrome-associated cardiomyopathy. Cell Stem Cell. 2014;15:9-11.

59. Ye L, Wang J, Beyer AI, et al. Seamless modification of wild-type induced pluripotent stem cells to the natural CCR5 $\Delta 32$ mutation confers resistance to HIV infection. Proc Natl Acad Sci U S A. 2014;111(26):9591-6.

60. Xie F, Ye L, Chang JC, et al. Seamless gene correction of $\beta$ thalassemia mutations in patient specific iPSCs using CRISPR/ Cas9 and piggybac. Genome Res. 2014;24:1526-33.

61. Gonzales F, Zhu Z, Shi ZD, et al. An iCRISPR platform for rapid multiplexable and inducible genome editing in human pluripotent stem cells. Cell Stem Cell. 2014;15(2):215-26.

62. Senis E, Fatouros C, Grosse S, et al. CRISPR/Cas9-mediated genome engineering: an adeno-associated viral (AVV) vector toolbox. Biotechnol J. 2014;9(11):1402-12.

63. Fu Y, Foden JA, Khayter C, et al. High-frequency off-target mutagenesis induced by CRISPR-Cas nucleases in human cells. Nat Biotechnol. 2013;31:822-6.

64. Ding Q, Regan SN, Xia Y, et al. Enhanced efficiency of human pluripotent stem cell genome editing through replacing TALENs with CRISPRs. Cell Stem Cell. 2013;12:393-4.

65. Veres A, Gosis BS, Ding Q. Low incidence of off-target mutations in individual CRISPR-Cas 9 and TALEN targeted human stem cell clones detected by whole-genome sequencing. Cell Stem Cell. 2014;15(1):27-30. Performs whole genome analysis to track any off-target events by TALENS/ CRISPRS 